\title{
Accurate (In)Direct Detection Rates for Neutralinos
}

\author{
Mia Schelke \\ Department of Physics, AlbaNova, Stockholm University, SE-106 91 \\ Stockholm, Sweden \\ Joakim Edsjö \\ Department of Physics, AlbaNova, Stockholm University, SE-106 91 \\ Stockholm, Sweden \\ Piero Ullio \\ SISSA, via Beirut 4, I-34014 Trieste, Italy
}

\begin{abstract}
Neutralinos arise as natural cold dark matter candidates in many supersymmetric extensions of the Standard Model of particle physics. If Galactic neutralinos exist, they could in principle be detected by both direct and indirect detection techniques. We use the DarkSUSY package to make very accurate calculations of the expected detection rates for a wide scan of parameter values. We compare the calculations with the sensitivity of current and future detectors, and conclude that some of the models are above the sensitivity limits. Comments are also made on the mutual dependence between the halo profile and the detection rates.
\end{abstract}

\section{Introduction}

Neutralinos $\left(\chi^{0}\right)$ are weakly interacting massive particles (WIMPs) which exist in supersymmmetric physics models (models which are theoretically well motivated, but have not been experimentally proved). The $\chi^{0}$ 's are often predicted to be stable, and are therefore candidates for the non-baryonic cold dark matter in the Universe (see e.g., Jungman, Kamionkowski, \& Griest (1996) for a review on supersymmetry and dark matter). All supersymmetric models have a number of free parameters. Different choices of parameter values can lead to vastly different predictions for the relic neutralino abundance and detection rates. The negative results from accelerator experiments, where one hopes to create supersymmetric particles, have already put strong constraints on the parameters. Even more severe are the constraints from cosmological observations on dark matter in general. The predicted relic density has to fall within the tiny interval that agrees with the observations. Our DarkSUSY package (Gondolo et al. 2002) makes very accurate calculations of the relic $\chi^{0}$ density, e.g., including all kinds of so-called co-annihilations. We can also hope to get a discovery or some constraints from some of the many ongoing and planned detectors which try to get an (in)direct glimpse of the neutralinos which are expected to move around in the Galactic halo. The supersymmetric parameters are not the only 
input needed to calculate the possible detection rates. It also depends highly on things such as the dark matter halo profile. In particular, a singular or clumpy halo profile can enhance the signal. The input of unknown astronomical models is a potential problem but also a great possibility. The search for galactic neutralinos can put some bounds on the combination of supersymmetric parameters and astronomical models, but it can be hard to get any stringent bound on them separately. The most fortunate situation would be to discover a supersymmetric signal by a number of techniques which are complementary to each other in such a way that they can pin down one particular supersymmetric model independently of the halo profile. The discovery could then, as well, be used to select the correct halo profile.

\section{Direct and indirect detection}

In direct detection one tries to measure the energy gained by elastic scattering of galactic neutralinos on the detector material. Just as Galactic neutralinos occasionally scatter on detector material, they will also from time to time scatter on the material in the Sun and Earth. After numerous scatterings, the neutralino has lost so much energy that it can no longer escape the Sun/Earth, thus suggesting that there is an accumulation of neutralinos in the centre of astronomical objects like the Sun and the Earth. The rate of neutralino annihilations will be correspondingly enhanced. There is a chance of making an indirect detection of the neutralino annihilation as some of the annihilation products are highly energetic neutrinos. We can also hope to make an indirect detection of neutralinos annihilating in the halo. Some detectors search for the expected continuous spectra of positrons, antiprotons and photons. Finally, one of the most prominent indirect signatures of supersymmetric dark matter is the expected gamma ray line of energy equal to the mass of the neutralino. It is produced in loop suppressed neutralino annihilations.

In the work in progress, on which we report in this paper, we use the DarkSUSY package (Gondolo et al. 2002) to make very accurate predictions for all kinds of direct and indirect detection rates. When we apply the minimal supergravity (supersymmetric) model, the Navarro-Frenk-White halo profile and assume no clumps, we find that the detection rates are in general quite small. In particular, the direct detection rates and the gamma ray line lie below the sensitivity limit of the current detectors. Fortunately, all kinds of planned detectors will cover a considerable part of the supersymmetric parameter space, although the main part will still remain unrevealed.

\section{References}

Gondolo, P., Edsjö, J., Ullio, P., Bergström, L., Schelke, M., \& Baltz, E. A. 2002, in idm2002 Conf. Proc. (astro-ph/0211238)

\& http://www.physto.se/ edsjo/darksusy/

Jungman, G., Kamionkowski, M., \& Griest, K. 1996, Phys. Rep., 267, 195 